\title{
Lepton Number Violating Signals of the Top Partners in the Left-Right Twin Higgs Model
}

\author{
Hock-Seng Goh \\ Department of Physics, University of California, Berkeley, CA \\ Christopher A. Krenke \\ Department of Physics, University of Maryland, College Park, MD 20742 and \\ Department of Physics, University of Arizona, Tucson, AZ 85721
}

\begin{abstract}
We study the collider signatures of the left-right twin Higgs model in the case that the right-handed neutrino mass is less than the mass of the right-handed gauge boson. In this scenario, new leptonic decay chains open up, allowing the particles which cancel the one-loop quadratic divergences of the Higgs, the right-handed gauge bosons and top-partners, to be discovered. Half of these events contain same-sign leptons without missing energy, which have no genuine standard model background and for which the backgrounds are purely instrumental. These signals may be used to complement other collider searches, and in certain regions of parameter space, may be the only way to observe the particles responsible for natural electroweak symmetry breaking in the left-right twin Higgs model.
\end{abstract}




\section{INTRODUCTION}

The standard model (SM) with a fundamental Higgs field suffers from an extreme sensitivity to short distance physics. If the cutoff of the SM is taken to be the Planck scale, this sensitivity leads to a tremendous fine tuning of the dimensionful parameters in the Higgs potential and a large hierarchy between the weak and Planck scales. If the cutoff of the SM is taken to be about $5 \mathrm{TeV}$, the minimum allowed by precision electroweak data, an unnatural adjustment of parameters persists and results in a "little hierarchy" 迎. This fact implies that new physics should exist at the $\mathrm{TeV}$ scale which is responsible for resolving the little hierarchy problem. This is an important observation, as the Large Hadron Collider (LHC) at CERN has been built with the aim of detecting particles with $\mathrm{TeV}$ scale masses. Therefore, new physics that is tightly connected to the nature of electroweak symmetry breaking is expected to be within the reach of the LHC. The nature of the new physics that cures the hierarchy problem, or the little hierarchy problem if the ultraviolet cutoff is taken to be less than $5 \mathrm{TeV}$, is highly constrained. Electroweak precision measurements have imposed very strong bounds on any new physics around a $\mathrm{TeV}$. These constraints pose a great challenge to designing models meant to address the little hierarchy problem.

One class of theories that address the little hierarchy problem is known as the little Higgs [2]. In these models, the SM Higgs doublet is a pseudo-Nambu-Goldstone boson (PNGB) of some spontaneously broken approximate global symmetry in which the SM $S U(2)$ electroweak symmetry is embeded [5, 6]. The Higgs mass vanishes at tree level due to shift symmetry, but will be generated by radiative corrections when interactions that break the global symmetry, such as gauge and Yukawa interactions, are included. At one-loop, multiple approximate global symmetries restrict the form of the quadratic terms in the Higgs potential such that together they form an invariant of the full global symmetry. Therefore, the one loop Higgs mass is only logarithmically sensitive to UV physics. This class of models is able to stabilize the elctroweak scale against the UV cutoff up to a scale of about $5-10 \mathrm{TeV}$.

Another class of theories that solves the little hierarchy problem by identifying the Higgs as a PNGB are twin Higgs models [7 9]. Instead of protecting the Higgs mass from receiving large radiative corrections by using several approximate global symmetries, twin Higgs theories use a discrete symmetry in combination with an approximate global symmetry to eliminate the quadratic divergences that arise at loop level. Together with the gauge symmetries of the model, the discrete symmetry mimics the effect of a global symmetry, thus stabilizing the Higgs mass.

In the left-right Twin Higgs model [8], the SM gauge symmetry is extended to $S U(2)_{L} \times$ 
$S U(2)_{R} \times U(1)_{B-L}$ 10], which is embedded into a global $U(4)$ symmetry. The Higgs arises as a PNGB when the $U(4)$ symmetry is spontaneously broken to $U(3)$. An additional $Z_{2}$ "twin symmetry" ensures that the quadratic terms in the Higgs potential have an accidental $U(4)$ symmetry. Since $U(4)$ invariant terms cannot contribute to the potential for the Goldstones, the Higgs is protected from receiving quadratically divergent contributions to its mass parameter. To evade precision electroweak bounds on $S U(2)_{R}$ gauge bosons without significantly affecting naturalness, an additional Higgs field, $\hat{H}$, is introduced that transforms as a fundamental under a second global $U(4)$ symmetry. This addition makes the approximate global symmetry of the theory $U(4) \times U(4)$. The new global symmetry does not significantly alter the form of the SM Higgs potential, allowing electroweak symmetry breaking to still happen naturally.

To identify the twin mechanism it is important to observe the heavy top quark partner, $T_{H}$, and the right-handed gauge boson, $W_{R}$. For a reasonable choice of parameters, the most straightforward way to observe both of these particles involves decays of the heavy top quark, which has a channel containing final state leptons that can be used as a trigger. It may be possible to reconstruct these events and observe the heavy top quark at the LHC [11]. However, this decay channel depends on a free parameter $M$, which could be very small or zero. In this limit, the heavy top quark can only decay hadronically [11], making these particles very difficult to observe at the LHC due to the large QCD background.

In this paper, we study an alternative way to observe the heavy top quark and the right-handed gauge boson. If a $\mathrm{TeV}$ scale right-handed Majorana neutrino is realized in the left-right twin Higgs model such that $m_{\nu_{R}}<m_{W_{R}}$, new leptonic channels open up that may allow detection of $W_{R}$ and $T_{H}$ at the LHC. Moreover, because the right-handed neutrino is Majorana, half of these decays are lepton number violating same-sign dilepton events without missing energy, which has no genuine SM background. If $M$ is small or zero, these lepton number violating signals may be the only way to observe the heavy top quark and the right-handed gauge boson at the LHC.

This paper is organized as follows: In section II, we review the left-right twin Higgs model and discuss its phenomenology in the decoupling case where the parameter $M$ is set to zero. In section III, we implement neutrino masses into the model and discuss a TeV scale right-handed neutrino. We study the collider phenomenology of the model in section IV, focusing on searches for the right-handed gauge boson, $W_{R}$, and the heavy top partner, $T_{H}$. We then conclude in section V. 


\section{LEFT-RIGHT TWIN HIGGS MODEL}

\section{A. Matter Content}

The fermionic content of the left-right twin Higgs model contains three generations of

$$
\begin{aligned}
& Q_{L}=(u, d)_{L}=(\mathbf{2}, \mathbf{1}, \mathbf{1} / \mathbf{3}) \quad L_{L}=(\nu, e)_{L}=(\mathbf{2}, \mathbf{1}, \mathbf{- 1}) \\
& Q_{R}=(u, d)_{R}=(\mathbf{1}, \mathbf{2}, \mathbf{1} / \mathbf{3}) L_{R}=(\nu, e)_{R}=(\mathbf{1}, \mathbf{2}, \mathbf{- 1}),
\end{aligned}
$$

where the square brackets indicate the quantum numbers of the corresponding fields under the $S U(2)_{L} \times S U(2)_{R} \times U(1)_{B-L}$ gauge symmetry of the theory. We see that in addition to the SM fermions, the theory includes right-handed neutrinos as required by left-right symmetry. There are two sets of Higgs fields which have quantum numbers 12, 13]

$$
\begin{array}{ll}
H_{L}=(\mathbf{2}, \mathbf{1}, \mathbf{1}) & H_{R}=(\mathbf{1}, \mathbf{2}, \mathbf{1}) \\
\hat{H}_{L}=(\mathbf{2}, \mathbf{1}, \mathbf{1}) & \hat{H}_{R}=(\mathbf{1}, \mathbf{2}, \mathbf{1}) .
\end{array}
$$

The reason for introducing the extra set of Higgs fields $\hat{H}$ is to satisfy precision electroweak constraints on $S U(2)_{R}$ gauge bosons. These constraints require the symmetry breaking scale $f$ of $S U(2)_{R}$ to be larger than about $2 \mathrm{TeV}$ 14. However, for this value of $f$, contributions to the Higgs potential from the top sector are very large since the top Yukawa is order one. This effect tends to reintroduce fine tuning to the model, destabilizing the weak scale. By adding an additional Higgs field $\hat{H}$, which acquires a vev $\langle\hat{H}\rangle=\hat{f} \sim 2 \mathrm{TeV}$ and does not couple to fermions, precision electroweak constraints on $S U(2)_{R}$ gauge bosons can be satisfied without affecting the top sector. This arrangement can be justified by imposing a discrete symmetry under which $\hat{H}$ is

odd while all other fields are even. This symmetry allows $\hat{H}_{L}$ to be stable, making it a natural dark matter candidate. It has been shown that this can account for the observed relic abundance of dark matter 15.

The Higgs potential is assumed to have an approximate $U(4) \times U(4)$ symmetry of which the $S U(2)_{L} \times S U(2)_{R} \times U(1)_{B-L}$ sub-group is gauged. After breaking the global $U(4)$ and the gauged $S U(2)_{R}$ symmetries, the SM Higgs doublet, which is among the NGBs, has no potential at tree level. However, a potential for the Higgs will be radiatively generated at one loop. In this scenario, both the mass and the quartic coupling of the Higgs are loop suppressed. To further reduce fine tuning, a tree level Higgs quartic can be introduced without generating a corresponding tree level mass term for the Higgs, as discussed in [9]. Since the Higgs potential is not relevant to our 
discussion of neutrino masses or collider signals, we shall not go into a detailed discussion of the Higgs potential.

The down-type Yukawa couplings of the SM emerge from non-renormalizable couplings of the form

$$
\left(\frac{\bar{Q}_{R} H_{R} H_{L}^{\dagger} Q_{L}+\bar{L}_{R} H_{R} H_{L}^{\dagger} L_{L}}{\Lambda}\right)+\text { h.c. }
$$

while the up-type Yukawa couplings of the SM emerge from non-renormalizable couplings of the form

$$
\left(\frac{\overline{Q_{R}} H_{R}^{\dagger} H_{L} Q_{L}}{\Lambda}\right)+\text { h.c. }
$$

When the field $H_{R}$ acquires a VEV of order $f$ breaking $S U(2)_{R} \times U(1)_{B-L}$ down to $U(1)_{Y}$, these non-renormalizable couplings reduce to the familiar Yukawa couplings of the SM. Unfortunately, this method of generating SM Yukawa couplings does not work well in the top sector since the top Yukawa coupling is order one. This problem is remedied by introducing the following vector like quarks, which transform as

$$
T_{L}=(\mathbf{1}, \mathbf{1}, \mathbf{4} / \mathbf{3}) \quad T_{R}=(\mathbf{1}, \mathbf{1}, \mathbf{4} / \mathbf{3})
$$

under $S U(2)_{L} \times S U(2)_{R} \times U(1)_{B-L}$. We can then write the following left-right symmetric interactions

$$
\left(y \bar{Q}_{R} H_{R}^{\dagger} T_{L}+y \bar{Q}_{L} H_{L}^{\dagger} T_{R}+M \bar{T}_{L} T_{R}\right)+\text { h.c. }
$$

The right-handed top quark of the SM then emerges as a linear combination of $T_{R}$ and the third generation up-type quark in $Q_{R}$, while the orthogonal linear combination is heavy. Provided $M \lesssim f$ and $y$ is of order one the physical top Yukawa will then also be of order one.

The parameter $M$ controls the mixing of the left-handed top with the $\mathrm{SU}(2)_{L} \operatorname{singlet} T_{L}$, and is therefore constrained by $Z \rightarrow b \bar{b}$. However, nothing prevents $M$ from simply being set to zero and therefore this is not a particularly tight constraint. However, the collider phenomenology of this model will depend on the size of this parameter. As we will see below, when $M$ is small, with $M=0$ as a extreme case of this scenario, the heavy top becomes difficult to observe in a hadron collider since it decays dominantly into an all jet final state.

\section{B. Phenomenology}

The left-right twin Higgs contains many new particles which may be observable at the LHC. The new particles include the right-handed gauge bosons $W_{R}$ and $Z_{R}$, a heavy top quark $T_{H}$, a 
right-handed neutrino $\nu_{R}$, and the Higgses $\hat{h}^{T}=\left(\hat{h}^{+}, \hat{h}^{0}\right), \phi^{ \pm}$and $\phi^{0}$. The gauge boson masses depend on the larger vev $\hat{f}$ and range from about $1-4 \mathrm{TeV}$, while the heavy top is typically lighter, ranging from $0.5-1 \mathrm{TeV}$. The $\phi^{0}$ mass depends on a free parameter in the theory, but is usually taken to be about $100 \mathrm{GeV}$. The charged Higgs $\phi^{ \pm}$mass ranges from about $200-400 \mathrm{GeV}$, while the $\hat{h}$ mass ranges from about $300 \mathrm{GeV}$ to $1 \mathrm{TeV}$. The right-handed neutrino mass arises from the operator

$$
\left(\frac{L_{R} \hat{H}_{R} \hat{H}_{R} L_{R}+L_{L} \hat{H}_{L} \hat{H}_{L} L_{L}}{\Lambda}\right)
$$

and is of order $\hat{f}^{2} / \Lambda$, which is about $1.5 \mathrm{TeV}$ for $\hat{f} \sim 4 \mathrm{TeV}$ and $\Lambda \sim 10 \mathrm{TeV}$. We will have more to say about neutrino masses in section [II]

What are the collider signatures of this model? The $Z_{R}$ decays to leptons providing a very clean signal, which may be observable at the LHC [11]. Detection of $W_{R}$ however, is more subtle. For now assume that $m_{\nu_{R}}>m_{W_{R}}$ and leptonic decays of the $W_{R}$ are kinematically forbidden. This was the scenario studied in [11]. In this case, the $W_{R}$ decays a large fraction of the time $(20 \%-$ $30 \%$ ) to a heavy top and a $b$-jet. Therefore the discovery potential of both the heavy top and $W_{R}$ depend critically on how the heavy top decays. As discussed in [11], the heavy top is produced in association with a $b$-quark, with a production cross section of about $500 \mathrm{fb}$. For a reasonable choice of $M=150 \mathrm{GeV}, T_{H}$ decays most often to $\phi^{ \pm} b$. For this value of $M$, the $\phi^{ \pm}$then decays mostly to $t b$. It is then possible to trigger on the leptonic decay of the top, giving the following decay chain

$$
T_{H} \rightarrow \phi^{ \pm} b \rightarrow t b b \rightarrow W b b b \rightarrow l \nu b b b .
$$

This scenario has been studied and shown to be detectable at the LHC with total luminosity of $10 \mathrm{fb}^{-1}[1]$.

$$
M=0: \text { The Dark Side of the Model }
$$

In the previous section, we reviewed the phenomenology of the left-right twin Higgs model when $m_{\nu_{R}}>m_{W_{R}}$ and for a small but reasonable value of $M=150 \mathrm{GeV}$. In this case, the decays of the heavy top included a leptonic final state, which could be used as a trigger. However, the phenomenology changes significantly when $M$ is very small, less than about $10 \mathrm{GeV}$. The crucial difference in this case is the decay of $\phi^{ \pm}$, which previously decayed to a SM top quark which then decayed leptonically. When $M=0$, the $\phi^{ \pm}$decays purely to charm and strange, leading to an all jet final state for heavy top decay. 

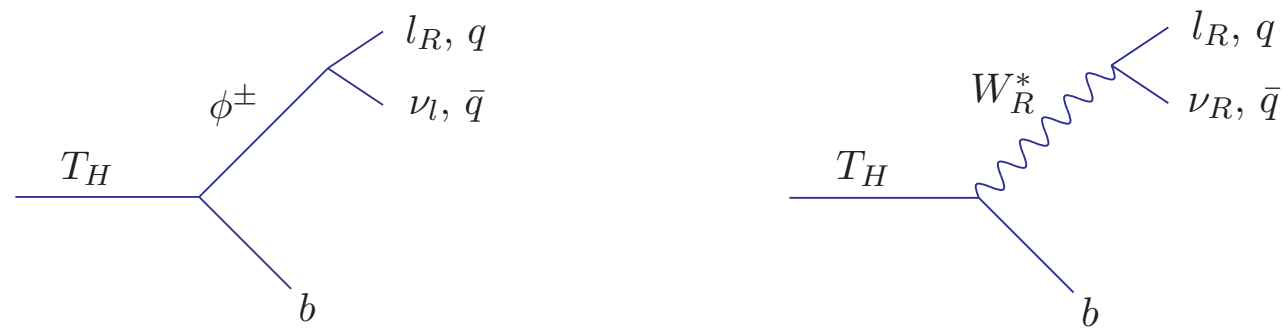

FIG. 1: Possible decays of the heavy top in the limit that $M=0$.

The reason $\phi^{ \pm}$does not decay to the SM top quark can be understood as follows. If $\phi^{ \pm}$is thought of as the charged component of $H_{R}$, then $\phi^{ \pm}$couples directly only to $b_{R}$ and $T_{L}$, which in the limit that $M=0$ are identified as the right-handed SM $b$ quark and the left-handed heavy top, respectively. When $M \neq 0$, mixing between $T_{L}$ and $T_{R}$ induces a coupling to the SM top quark that is proportional to $M / f$ for $M<<f$. Therefore, in the limit $M \rightarrow 0, \phi^{ \pm}$cannot decay to a SM top quark.

What about other decay channels for $T_{H}$ ? Two other decay channels are possible and are shown in Fig. 1. In the first decay channel, $\phi^{ \pm}$will dominantly decay to $q \bar{q}$ because the leptonic decay channel is suppressed by the neutrino Yukawa coupling constant. In the second decay channel, $\nu_{R}$ is kinematically unavailable and therefore the leptonic channel is only available through an off shell $\nu_{R}$, which is highly suppressed. Therefore, in the limit that $M \rightarrow 0, T_{H}$ can only decay hadronically, leading to an all-jet final state for heavy top decay. Detection of the heavy top at the LHC then becomes difficult. In this scenario, the model may become one of those in which the true mechanism of natural electroweak symmetry breaking is beyond the reach of the LHC.

Since the small $M$ parameter space is large, technically natural, and does not affect the twin mechanism, it is important to examine this possibility more closely. The hope lies in the size of right-handed neutrino mass relative to the mass of the $W_{R}$. If $m_{\nu_{R}}<m_{W_{R}}$, leptonic decay channels of the heavy top open up and provide a way to observe the heavy top partner that is independent of the parameter $M$. As a preliminary, we discuss neutrino mass generation in the left-right twin Higgs model in the next section. 


\section{NEUTRINO MASS: SEESAW AT THE TEV SCALE}

There is more than one way to implement neutrino mass in the left-right twin Higgs model. For a detailed study of neutrino mass generation in this context, see [16]. If lepton number is a good symmetry of the theory, the neutrino masses must be Dirac. In this case, the smallness of the neutrino masses can be understood purely as a result of their small Yukawa couplings. If lepton number is not conserved, the neutrino masses can be Majorana. In this case, the lightness of the SM neutrinos can be understood as a result of the seesaw mechanism [17. We will assume that left-right symmetry is exact in the neutrino sector as in all other sectors of the model. The most general collection of operators that generate neutrino masses are the following: Dirac neutrino masses arise from the operators

$$
\begin{aligned}
y_{\nu}\left(\frac{L_{R} H_{R} H_{L} L_{L}}{\Lambda}\right)+\text { h.c. } & \rightarrow y_{\nu} \frac{f v}{\Lambda} \nu_{L} \nu_{R}+\text { h.c. } \\
& =m_{D} \nu_{L} \nu_{R}+\text { h.c. }
\end{aligned}
$$

while the operators

$$
\begin{aligned}
& y_{1}\left(\frac{L_{R} \hat{H}_{R} \hat{H}_{R} L_{R}+L_{L} \hat{H}_{L} \hat{H}_{L} L_{L}}{\Lambda}\right)+\text { h.c. } \rightarrow y_{1} \frac{\hat{f}^{2}}{\Lambda} \nu_{R} \nu_{R}+\text { h.c. } \\
& y_{2}\left(\frac{L_{R} H_{R} H_{R} L_{R}+L_{L} H_{L} H_{L} L_{L}}{\Lambda}\right)+\text { h.c. } \rightarrow y_{2}\left(\frac{f^{2}}{\Lambda} \nu_{R} \nu_{R}+\frac{v^{2}}{\Lambda} \nu_{L} \nu_{L}\right)+\text { h.c. }
\end{aligned}
$$

generate Majorana masses for the right-handed neutrinos, $\nu_{R}$, and the left handed neutrinos, $\nu_{L}$. One possibility is that we assume lepton number is not violated. In this case, the operators in eq. (10) and eq. (11) are not present. The light neutrinos, $\nu=\left(\nu_{L}, \bar{\nu}_{R}\right)$, are Dirac fermions and the small neutrino masses are just the result of small Yukawa couplings, which are around $10^{-12}$. The other possibility is that light Majorana neutrinos are generated through a TeV scale seesaw mechanism. If we no longer assume lepton number conservation, all the operators above are allowed. Since the operator in eq. (11) gives both $\nu_{R}$ and $\nu_{L}$ a Majorana mass, this term should be small, i.e. $y_{2}<10^{-11}$. Note that eq. (10) does not generate a Majorana mass for $\nu_{L}$ because $\left\langle\hat{H}_{L}\right\rangle=0$. The SM neutrinos can then obtain a Majorana mass of the right size if the coupling constant $y_{\nu}$ is

$\sim 10^{-5}$, which is of order the electron Yukawa coupling. We will follow this possibility from now on and assume $y_{2}=0$. A $Z_{4}$ symmetry where $H$ is neutral may be used to justify this possibility. 


\section{Constraints on Majorana Right-handed Neutrinos}

Right-handed neutrinos with masses of order a TeV have been studied by several authors [18]. Since there are three generations of right-handed neutrinos, the details of experimental constraints and their collider signals depend substantially on their mass differences and mixing angles. For simplicity, we do not consider the most general mass matrix and assume a nearly degenerate mass spectrum.

What are the constraints on right-handed neutrinos? Big Bang nucleosynthesis (BBN) puts severe constraints on new light degrees of freedom. However, particles that are heavier than an $\mathrm{MeV}$ and which do not decay during the era of BBN are completely free of this constraint. Another bound comes from tritium decay, but that also only constrains light particles with masses less than about an $\mathrm{MeV}$. There are stronger restrictions on massive right-handed neutrinos from precision measurements of $Z$-decay and single $\nu_{R}$ production [19, 20]. However, we will only consider righthanded neutrino masses of order a few hundred $\mathrm{GeV}$, which are also free from these constraints.

If the right-handed neutrino is Majorana, the most stringent bound on its mass arises from neutrinoless double beta decay. The bound can be approximately expressed in terms of the bound on the light neutrino effective mass $m_{e e}^{m a x}$ as in 21]

$$
\frac{m_{\nu_{R}}}{p^{2}-m_{\nu_{R}}^{2}} \prod_{i=1,2} \frac{V_{i, q} V_{i, e}}{g_{2}^{2}}\left(\frac{m_{W}^{2}}{m_{x i}^{2}}\right) \leq \frac{m_{e e}^{\max }}{m_{e} m_{p}} \frac{1-\chi_{F}}{\chi_{H}},
$$

where $m_{x i}$ are masses of the particles that mediate beta decay and $V_{i, q}$ and $V_{i, e}$ are the corresponding couplings to the quarks and the electron, respectively. $p$ is the typical energy exchanged in the process, which is of order $100 \mathrm{MeV} \cdot \chi_{F}$ and $\chi_{H}$ are the nuclear matrix elements corresponding to exchanging $\nu_{L}$ and $\nu_{R}$, respectively, and are given in [21]. For example, in an extension of the SM with only right-handed neutrinos and the seesaw mechanism, $x_{i}=W^{ \pm}$and

the couplings are $V_{i, e} \sim g_{2} \delta_{s s}$ where $\delta_{s s}$ is the seesaw mixing factor, $\delta_{s s} \sim \frac{m_{D}}{m_{\nu_{R}}} \sim \sqrt{\frac{m_{\nu}}{m_{\nu_{R}}}}$. For a TeV scale right-handed neutrino, $\delta_{s s} \sim 10^{-7}$. In general, several diagrams may contribute to neutrinoless double beta decay and various parameters will be constrained by experiment. In the case of the left-right twin Higgs model, the diagrams with standard model $W$ exchange contain the seesaw mixing factor and, therefore, are much more suppressed than those with $W_{R}$ exchange, which are only suppressed by the mass of the $W_{R}$. Other subdominant diagrams with charged Higgs exchange are also suppressed. The contribution from $W_{R}$ to neutrinoless double beta decay therefore leads to the tightest constraint. Using the experimental bound given in [22] and eq. (12]), 
we find

$$
m_{\nu_{R}} m_{W_{R}}^{4} \geq 1.56 \mathrm{TeV}^{5}
$$

The lower bound of the right-handed neutrino mass is then

$$
m_{\nu_{R}} \geq 120\left(\frac{1.9 \mathrm{TeV}}{m_{W_{R}}}\right)^{4} \mathrm{GeV}
$$

which is well below the range of mass we will be considering. We will from now on treat the neutrino mass, $m_{\nu_{R}}$, as a free parameter and study its collider phenomenology.

\section{IV. $M=0$ PHENOMENOLOGY}

The phenomenology of the left-right twin Higgs model has been studied by many authors [11, 23]. We will focus on the limit where the top mixing parameter, $M$, is set to zero. When $M=0$ and the right-handed neutrino is heavier than $W_{R}$, some of the new particles including $W_{R}$ and heavy top partner $T_{H}$ are difficult to detect because their decay channels are dominated by hadronic final states. Here we consider the limit when the right-handed neutrino mass is less than the mass of $W_{R}$. The search for $W_{R}$ will then be much more effective due to the opening of leptonic decay channels. Even better, the leptonic decay has a $50 \%$ chance of violating lepton number due to the Majorana nature of $\nu_{R}$. The same advantages will also apply to $T_{H}$, but these searches depend on the mass of $\nu_{R}$ relative to the mass of $T_{H}$. There are two possibilities: (i) $m_{\nu_{R}}<m_{T_{H}}$ and (ii) $m_{\nu_{R}}>m_{T_{H}}$, which we consider separately. In the following analysis we choose the following typical parameter set: $f=800 \mathrm{GeV}$, which implies $\hat{f} \approx 4 \mathrm{TeV}, m_{W_{R}} \approx 1.9 \mathrm{TeV}$ and $T_{H} \approx 780 \mathrm{GeV}$ for a reasonable choice of soft parameters. A different choice of soft parameters will lead to a different set of masses, so these are not strict mass relations. However, this will not qualitatively affect our conclusions. Let us begin with a discussion of the search for $W_{R}$, which can be done independently from $T_{H}$.

\section{A. $W_{R}$ Search}

The $W_{R}$ is dominantly produced via a Drell-Yan process and subsequently decays leptonically to $\nu_{R}+l^{ \pm}$with a branching fraction of about a 10\%. $\nu_{R}$ then decays to $l^{ \pm}+X$ through an off shell $W_{R}$ or an on shell charged Higgs $\phi^{ \pm}$, as shown in Fig. 2. Here, $X$ represents any number of final state jets. Due to the fact that $\phi^{ \pm}$only decays hadronically and the leptonic decays of the off shell

$W_{R}$ are kinematically forbidden, $X$ cannot contain any leptons. As argued in the section IIB, this 


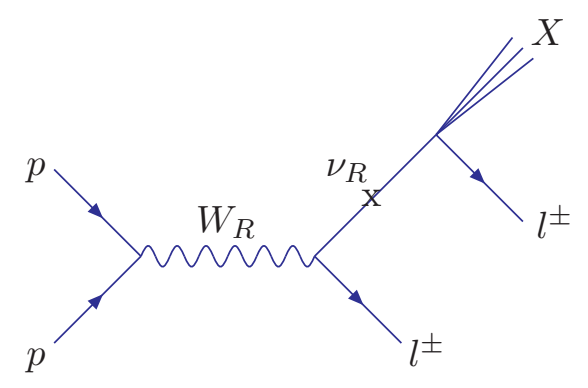

FIG. 2: Diagrammatic view of $W_{R}$ production and its lepton number violating decay channel.
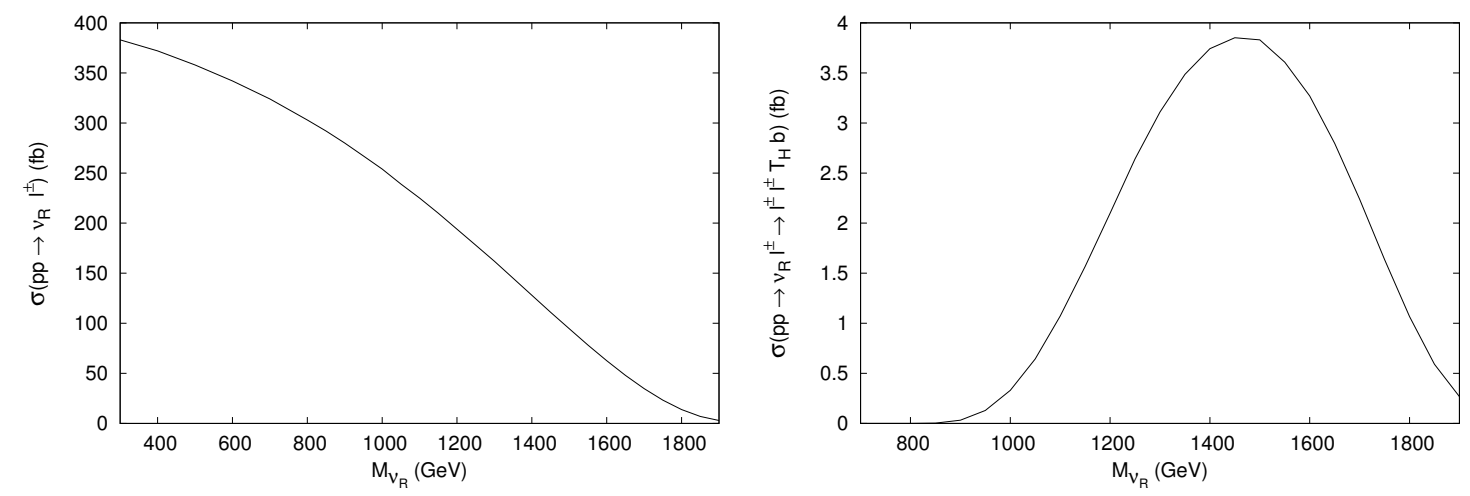

FIG. 3: The left plot shows the production cross section of the right handed neutrino $\nu_{R}+l^{ \pm}$with an associated lepton $\left(e^{ \pm}\right.$or $\left.\mu^{ \pm}\right)$as a function of $m_{\nu_{R}}$. The right plot shows the production cross section of the heavy top $T_{H}$ through the decay of $\nu_{R}$ in association with same-sign leptons ( $e^{ \pm}$or $\left.\mu^{ \pm}\right)$as a function of $m_{\nu_{R}}$.

is precisely why the heavy top decays purely hadronically in the decoupling limit when $M \rightarrow 0$. Since $\nu_{R}$ is Majorana, half of these events will contain same-sign leptons. The signal is therefore same-sign dilepton $l^{ \pm} l^{ \pm} X$ events without missing energy, which has no genuine SM background.

The production cross section at the LHC of $\nu_{R}+l^{ \pm}$, where $l=e, \mu$, is calculated using CalcHEP [24] and is shown in Fig. 3 as a function of the right-handed neutrino mass $m_{\nu_{R}}$. Half of these events will have same-sign dilepton without missing energy. For example, if $m_{\nu_{R}}=1 \mathrm{TeV}$, the production cross section is about $250 \mathrm{fb}$, which leads to approximately 3750 same-sign dilepton events with $30 \mathrm{fb}^{-1}$ of total luminosity. The invariant mass distribution of the final state particles should provide a clear signal of $W_{R}$. Furthermore, the invariant mass distribution of the jets plus one lepton should provide a signal of $\nu_{R}$.

As mentioned above, there is no genuine SM background for the same-sign dilepton signal and 
so the background at the LHC is purely instrumental. This mostly arises from a mismeasurement of missing energy and/or a lepton's charge. Another possibility is the misidentification of a jet as a lepton. Background events can therefore arise from any of the following processes:

$$
\begin{gathered}
p p \rightarrow l^{+} l^{-} j j \\
p p \rightarrow t \bar{t} \rightarrow l^{+} l^{-} b \bar{b}+\mathbb{E}_{T} \\
p p \rightarrow W^{ \pm} W^{ \pm} j j \rightarrow l^{ \pm} l^{ \pm} j j+\mathbb{E}_{T} \\
p p \rightarrow W^{+} W^{-} j j \rightarrow l^{+} l^{-} j j+\mathbb{E}_{T} \\
p p \rightarrow t \bar{t} \rightarrow l^{ \pm} l^{ \pm} b c j j+\mathbb{E}_{T} \\
p p \rightarrow t \bar{t} \rightarrow l^{ \pm} b \bar{b} j j+\mathbb{E}_{T} \\
p p \rightarrow j j j j \\
p p \rightarrow l^{ \pm} j j j+\mathbb{E}_{T}
\end{gathered}
$$

A complete analysis of these backgrounds requires a full detector simulation, which is beyond the scope of this paper. Here we estimate the upper bounds of these background processes using the parton level computation tool Madgraph/Madevent [25] to show the possible reach of the LHC. To approximate the detector simulation, we take the average performance of the ATLAS detector [26] to be

$$
\begin{aligned}
\epsilon_{l} & =0.85 \quad c_{l}=0.02 \\
R_{l, j} & =10^{-4} R_{l, b}=0.02,
\end{aligned}
$$

where $\epsilon_{l}$ is the lepton acceptance efficiency, $c_{l}$ is the probability of lepton charge misidentification, $R_{l, j}$ is the probability that a light jet will fake a lepton and $R_{l, b}$ is the probability that a $b$ jet will fake a lepton. To select events, we adopt the following basic kinematic acceptance cuts

$$
p_{T}^{l}>10 \mathrm{GeV}, \quad p_{T}^{j}>15 \mathrm{GeV}, \quad\left|\eta^{l, j}\right|<2.5
$$

First consider the background events due to $l^{+} l^{-} j j$ production at the LHC as in eq. (15). The cross section for this process at the LHC is dominated by the $Z j j$ cross section, which is approximately $10^{4} \mathrm{pb}$. After accounting for the leptonic decay of the $Z$ and the charge mismeasurement rate, the $l^{+} l^{-} j j$ background is approximately $40 \mathrm{pb}$, which is very large compared to the $W_{R}$ signal. However, a significant reduction of this background can be easily achieved by applying the following additional cuts: 


$$
\begin{array}{ll}
p_{T}^{\text {leading lepton }}>200 \mathrm{GeV}, & p_{T}^{\text {two jets }}>300 \mathrm{GeV}, \\
p_{T}^{\text {second lepton }}>100 \mathrm{GeV}, & m_{l l}>200 \mathrm{GeV} .
\end{array}
$$

The cut on the invariant mass of the two leptons is useful because the two background leptons are produced via $Z$ decay, while the signal leptons are produced at different steps in a cascade decay. Therefore, this cut forces the $Z$ to be off-shell, which suppresses the background substantially. After imposing these additional cuts and accounting for charge misidentification, the background cross section arising from $l^{+} l^{-} j j$ production is estimated to be about $0.05 \mathrm{fb}$.

Background events will also arise due to $t \bar{t}$ production in which both tops decay semi-leptonically as in eq. (16). After the basic acceptance cuts of eq. (25) and the extended cuts of eq. (26), we estimate the cross section for $t \bar{t} \rightarrow b \bar{b} l^{+} l^{-} \nu_{l} \bar{\nu}_{l}$ to be approximately $1.84 \mathrm{fb}$. After the mismeasurement of one lepton's charge is taken into account, this background reduces to about $0.074 \mathrm{fb}$.

The background from $W^{ \pm} W^{ \pm} j j$ production in eq. (17) contains same-sign leptons and therefore is not suppressed by the rate of charge misdentification. Note that we include both QCD and electroweak jet production in the process $p p \rightarrow W^{ \pm} W^{ \pm} j j$. After applying the cuts of eq. (25) and eq. (26), the $l^{ \pm} l^{ \pm} j j$ cross section due to $W^{ \pm} W^{ \pm} j j$ production is reduced to approximately $0.07 \mathrm{fb}$.

Another background arising from $W$ production is shown in eq. (18). This background does not contain same-sign leptons and is therefore suppressed by the charge misidentification rate. After applying the cuts and accounting for the probability of misidentifying the lepton's charge, we estimate this background cross section to be less than about $0.02 \mathrm{fb}$.

We next consider a second background arising from $t \bar{t}$ production, as shown in eq. (19). Assuming two of the jets in this process (light or $b$ ) have $p_{T}>300 \mathrm{GeV}$, we estimate the cross section for this process to be less than about $18 \mathrm{fb}$, which is quite large. However, since the lepton and charm quark are typically produced via the decay of a boosted $b$ meson, the two will be highly collimated. Therefore, in addition to the cuts of eq. (25) and eq. (26), we also require that the jet-lepton isolation, $\Delta R_{l j}$, be greater than $0.1^{*}$. Furthermore, we require that the invariant mass of

\footnotetext{
* Instead of studying the $b$ quark hadronization, shower and decay, we simplify the analysis by studying the $b$ decay at the parton level. However, the $b$ meson produced in this reaction is typically less energetic than the $b$ quark from the hard scattering because energy is carried away during fragmentation. In order to account for this difference, we consider the distribution of the scaled energy $x_{E}=E_{B} / E_{b}$ given in [27]. This distribution is peaked at $x_{E} \sim 0.8$. A numerical analysis shows that taking a fixed value of $x_{E}=0.8$ is a good approximation to the full distribution. We therefore use this scaled energy factor in our parton level analysis.
} 
all final state particles fall within $200 \mathrm{GeV}$ of the mass of the $W_{R}$ and the total missing $p_{T}$ carried by the neutrinos be less than $100 \mathrm{GeV}$. These cuts are very efficient and reduce the background of eq. (19) to less than $1.84 \times 10^{-3} \mathrm{fb}$.

In addition to producing a same-sign lepton via $b$ decay, it is also possible for a bottom quark in eq. (19) to fake a lepton, with a probability of about 1 in 50. To estimate this background, we apply the lepton $p_{T}$ cuts of eq. (26) to one of the $b$ jets and the jet $p_{T}$ cuts to two of the remaining three jets. After the cuts and accounting for the probability that a $b$ jet will fake a lepton, we find this background can be reduced to less than about $0.55 \mathrm{fb}$.

Additional backgrounds involving the misidentification of a jet as a lepton are shown in eqs. (21) and (22). We first consider the production cross section of $j j j j$ in which two jets are misidentified as leptons. To determine an upper bound on the cross section for this process, we first estimate di-jet production at the LHC, $p p \rightarrow j j$. For $p_{T}^{j}>300 \mathrm{GeV}$, the di-jet production cross section at the LHC is approximately $8.5 \times 10^{3} \mathrm{pb}$. To pass the extended cuts of eq. (26), all of the jets in the four-jet are required to be hard and have similar $p_{T}$. Logarithmically enhanced soft radiation will not be sufficient to produce the required high $p_{T}$ jets. Therefore, four-jet background events must arise from four partons with high $p_{T}$ and will be reduced by at least a factor of $\alpha_{s}^{2} \sim 0.015$ and two factors of the jet-lepton fake rate, $10^{-4}$. Therefore, we expect the background arising from four jet events to be suppressed by at least a factor of $10^{-10}$ compared to the di-jet production cross section. Since the cuts of eq. (26) require two jets to have $p_{T}>300 \mathrm{GeV}$, we estimate the background cross section arising from four jets can be at most the di-jet production cross section with $p_{T}^{j}>300 \mathrm{GeV}$ suppressed by $10^{-10}$. Therefore, we expect the cross section for background events arising from the misidentification of two light jets as leptons to be less than approximately $1.3 \times 10^{-3} \mathrm{fb}$.

This type of background can also arise from $b \bar{b} j j$ production. While this process is suppressed compared to that of $j j j j$ production, the probability for a $b$ jet to fake a lepton is significantly greater. However, since $b$ and $\bar{b}$ have opposite charge, this background is also suppressed by the probablility of misidentifying the $b$ quark's charge. After accounting for this suppression, the background arising from $b \bar{b} j j$ contributes only a few percent compared to that of $j j j j$ production. We therefore ignore this contribution in our analysis.

We next consider the process $p p \rightarrow l^{ \pm} \nu j j j$, in which one jet is misidentified as a lepton as in eq. (22). To estimate an upper bound on the cross section for this process, we first calculate the production cross section of $l^{ \pm} \nu j j$ requiring that both jets have $p_{T}>300 \mathrm{GeV}$ and the lepton has $p_{T}>100 \mathrm{GeV}$. We find this cross section to be approximately $1 \mathrm{pb}$. After accounting for 


\begin{tabular}{|l|r|r|}
\hline Process & $\sigma\left(p_{T}^{j}>300 \mathrm{GeV}\right)(\mathrm{fb})$ & $\sigma\left(p_{T}^{j}>200 \mathrm{GeV}\right)(\mathrm{fb})$ \\
\hline$l^{+} l^{-} j j$ & 0.05 & 0.16 \\
\hline$b \bar{b} l^{+} l^{-} \nu_{l} \bar{\nu}_{l}$ & 0.074 & 0.25 \\
\hline$W^{ \pm} W^{ \pm} j j$ & 0.07 & 0.13 \\
\hline$W^{+} W^{-} j j$ & 0.02 & 0.06 \\
\hline$l^{ \pm} l^{ \pm} b c \nu \nu j j$ & $1.8 \times 10^{-3}$ & $5.3 \times 10^{-3}$ \\
\hline$l^{ \pm} b \bar{b} \nu j j$ & 0.55 & 2.4 \\
\hline$j j j j$ & $1.3 \times 10^{-3}$ & $8.1 \times 10^{-3}$ \\
\hline$l^{ \pm} \nu j j j$ & 0.05 & 0.11 \\
\hline \hline Total Background & $\approx 0.8$ & $\approx 3.1$ \\
\hline \hline Signal & 12.5 & 17 \\
\hline
\end{tabular}

TABLE I: A summary of the dominant backgrounds to the lepton number violating final state $l^{ \pm} l^{ \pm} j j$ arising from the decay of the right-handed $W_{R}$. For comparison, we also show the expected signal for $m_{\nu_{R}}=1.5 \mathrm{TeV}$.

the additional hard jet with a factor of $\alpha_{s}$ and the probability that a jet will fake a lepton, we find that the cross section for $l^{ \pm} l^{ \pm} j j$ arising from the misidentification of a jet as a lepton in the process $p p \rightarrow l^{ \pm} \nu j j j$ to be less than approximately $0.04 \mathrm{fb}$. As discussed previously, the light jets in this process may be replaced by $b$ jets. However, unlike the four-jet background, only one $b$ jet is required to fake a lepton. Therefore, there is no additional suppression due to misidentifying the charge of a $b$ quark. We estimate this effect to contribute at most an additional $0.01 \mathrm{fb}$ of background. Therefore, we expect the total background cross section arising from $l^{ \pm} \nu j j j$ to be less than approximately $0.05 \mathrm{fb}$.

The dominant backgrounds to the $l^{ \pm} l^{ \pm} j j$ signal described above are summarized in Table (西). For comparison, we also include in this table an additional set of background cross sections in which the $p_{T}$ cuts on the jets are taken to be $200 \mathrm{GeV}$. We estimate the total background in these two cases to be less than $0.8 \mathrm{fb}$ and $3.1 \mathrm{fb}$ for $p_{T}^{j}>300 \mathrm{GeV}$ and $p_{T}^{j}>200 \mathrm{GeV}$, respectively. For $m_{\nu_{R}}=1.5 \mathrm{TeV}$, the signal cross section is about $50 \mathrm{fb}$ before the cuts and is reduced to approximately $12.5 \mathrm{fb}(17 \mathrm{fb})$ after the cuts given that $p_{T}^{j}>300 \mathrm{GeV}(200 \mathrm{GeV})$. For the signal, we also take into account the lepton acceptance efficiency. More thorough cuts on missing energy and the invariant masses may further reduce these backgrounds. However, since the expected signal of $W_{R}$ and $\nu_{R}$ is already much larger than the background, we do not analyze these backgrounds any further. Therefore, in this scenario it should be possible to observe the right-handed gauge boson $W_{R}$ and the right-handed neutrino $\nu_{R}$ at the LHC. 


\section{B. $T_{H}$ Search: $m_{\nu_{R}}>m_{T_{H}}$}

In this case, $T_{H}$ decays solely to $b+\phi^{ \pm}$and results in an all jet final state. However, $T_{H}$ can also be produced by the decay of $\nu_{R}$ through an off shell $W_{R}$. The process is

$$
p p \rightarrow l^{ \pm} \nu_{R} \rightarrow l^{ \pm} l^{ \pm} b T_{H} \rightarrow l^{ \pm} l^{ \pm} b b j j
$$

with the cross section

$$
\sigma\left(p p \rightarrow T_{H} l^{ \pm} l^{ \pm} b\right) \approx \sigma\left(p p \rightarrow \nu_{R} l^{ \pm}\right) \times B r\left(\nu_{R} \rightarrow l^{ \pm} T_{H} b\right)
$$

which is a few $\mathrm{fb}$, as shown in Fig. 3. By requiring that the leptons be of the same-sign, applying kinematic cuts and possibly $b$-tagging, it should be possible to separate these events from background at the LHC. The invariant mass distribution of three of the four jets should provide a signal of $T_{H}$.

As with the same-sign $l^{ \pm} l^{ \pm} j j$ signal, the background for same-sign $l^{ \pm} l^{ \pm} b b j j$ signal is purely instrumental. To analyze the background, we implement kinematic cuts that are identical to those of the previous section except that we now require four hard jets,

$$
p_{T}^{\text {two leading jets }}>200 \mathrm{GeV}, \quad p_{T}^{\text {next two leading jets }}>80 \mathrm{GeV} .
$$

Since the $T_{H}$ signal contains at least four hard jets, background processes similar to those described in the previous section containing only two jets (eqs. (15)-(18) and eqs. (21)-(22)) should be suppressed by a factor of approximately $\alpha_{s}^{2}$. This factor accounts for the two additional hard partons required to mimic the signal. However, this argument does not apply to the events arising from $t \bar{t}$ production. To satisfy the cuts, the transverse momentum of the tops must be about $400 \mathrm{GeV}$ in order to decay to a $200 \mathrm{GeV}$ jet and lepton. This means a potentially large logarithm may be associated with the extra $80 \mathrm{GeV}$ jet production due to the large gap between 400 and $80 \mathrm{GeV}$. Therefore, the production cross section of $t \bar{t}$ plus jets (with $p_{T}>80 \mathrm{GeV}$ ) may not be suppressed compared to that of $t \bar{t}$ production.

The total background can be divided into two classes. One class arises from $t \bar{t}+X$ production, which is expected to be similar to that obtained in the $W_{R}$ search and is about $2.6 \mathrm{fb}$. The other class of background includes everything else, which is suppressed by $\alpha_{s}^{2}$ and is about $0.007 \mathrm{fb}$. The background is therefore dominated by $t \bar{t}+X$, which is about the size of the signal and needs further analysis.

The same-sign lepton, four-jet background arising from $t \bar{t}$ production, eq. (19), is small compared to the signal and therefore no further analysis is needed. However, the background arising from 


\begin{tabular}{|l|r|}
\hline Background process & $\sigma\left(p_{T}^{j}>200 \mathrm{GeV}\right)(\mathrm{fb})$ \\
\hline$b c l^{ \pm} l^{ \pm} \nu \bar{\nu} j j$ & 0.005 \\
\hline$b \bar{b} l^{ \pm} \nu j j j$ & 0.057 \\
\hline$b \bar{b} l^{+} l^{-} \nu \bar{\nu} j j$ & 0.004 \\
\hline$\alpha_{s}^{2}$ Suppressed & 0.007 \\
\hline \hline Total Background & $\approx 0.07$ \\
\hline \hline Signal & 0.87 \\
\hline
\end{tabular}

TABLE II: A summary of the dominant backgrounds to the lepton number violating final state $l^{ \pm} l^{ \pm} b b j j$ arising from the decay of a right-handed neutrino to a heavy top partner, $T_{H}$. For comparison, we also show the expected signal for $m_{\nu_{R}}=1.5 \mathrm{TeV}$.

a $b$ quark faking a lepton, eq. (20), and the leptonic decays of both tops, eq. (16) need further suppression. For these processes, we apply the same invariant mass and missing $p_{T}$ cuts as those used in the $W_{R}$ search. $t \bar{t} j$ and $t \bar{t} j j$ events are generated by MadEvent to estimate the background for eq. (20) and eq. (16). The above kinematic cuts are then imposed on the decay products of both of the tops. We find the cross section of eq. (20) to be about $0.057 \mathrm{fb}$ and that of eq. (16) to be about $0.004 \mathrm{fb}$.

The backgrounds contributing to the $l^{ \pm} l^{ \pm} b b j j$ signal are summarized in Table ([II). Given the cuts described above, we estimate the total background cross section of the $T_{H}$ signal to be about $0.07 \mathrm{fb}$. To predict the possible reach of the LHC, we must understand how the $l^{ \pm} l^{ \pm} b b j j$ signal is affected by the cuts discussed above. After the cuts, the $l^{ \pm} l^{ \pm} b b j j$ signal is reduced by about $80 \%$ when $m_{\nu_{R}}=1.5 \mathrm{TeV}$. This results in a cross section of approximately $0.87 \mathrm{fb}$ after accounting for the lepton acceptance efficiency. Clearly, the signal is significantly larger than the estimated background. With only $10 \mathrm{fb}^{-1}$ of total luminosity, we expect about 9 signal events while the SM prediction is less than one. However, due to the combinatoric ambiguity in the reconstruction of $T_{H}$, more events may be needed. It may also be possible to use kinematics to pick out the correct combination.

We stress that the procedure outlined above provides only a rough estimate of the relevant backgrounds. The true background could be larger by an $O(1)$ factor. On the other hand, a more detailed study involving missing energy cuts and a careful utilization of invariant mass cuts may result in a significant improvement in the signal to background ratio. This study, as well as the reconstruction of $T_{H}$, requires a more realistic analysis, which we leave for future work. Therefore, we conclude that it should be possible to detect the heavy top partner $T_{H}$ at the LHC, provided 
that $m_{\nu_{R}}>m_{T_{H}}$.

\section{C. $T_{H}$ Search: $m_{\nu_{R}}<m_{T_{H}}$}

To observe the leptonic decays of the heavy top in this case, we must look for the decays of $T_{H}$ to $\nu_{R}$. Once produced via $W_{R}$ decay, $T_{H}$ can decay to $\nu_{R}+b+l^{ \pm}$through an off shell $W_{R}$. However, there is another decay channel which does not involve $\nu_{R}, T_{H} \rightarrow \phi^{ \pm}+b$. As discussed above, $\phi^{ \pm}$decays to jets, so the signal is either $p p \rightarrow l l b b j j$ or $p p \rightarrow b b j j$. The cross sections for these processes are determined by the partial decay width of $T_{H}$ to these channels

$$
\begin{aligned}
\Gamma_{T_{H} \rightarrow \nu_{R} l^{ \pm} b} & \sim 10^{-5} \mathrm{GeV} \\
\Gamma_{T_{H} \rightarrow b \phi^{ \pm}} & \sim 3 \mathrm{GeV}
\end{aligned}
$$

As expected, the two-body decay dominates the decay width, making the branching fraction $\operatorname{Br}\left(T_{H} \rightarrow \nu_{R} b l^{ \pm}\right)$very small. The cross section for $p p \rightarrow T_{H} b \rightarrow b b l l j j$ is then about $10^{-3} \mathrm{fb}$, which is too small to be observed at the LHC. Therefore, in this case it will not be possible to detect the heavy top partner $T_{H}$.

\section{CONCLUSION}

In summary, we have shown that a $\mathrm{TeV}$ scale right-handed neutrino in the left-right twin Higgs model leads to interesting lepton number violating signatures for $W_{R}$ and $T_{H}$ at the LHC, provided that $m_{\nu_{R}}<m_{W_{R}}$. Lepton number violating decays of right-handed $W_{R}$ should be observable provided that $W_{R}$ and $\nu_{R}$ are not nearly degenerate. Detection of the heavy top is possible if $m_{\nu_{R}}>m_{T_{H}}$. These signals may be used to complement other collider searches for $W_{R}$ and $T_{H}$. In the limit that $M \rightarrow 0$, these signatures may be the only way to observe the particles responsible for natural electroweak symmetry breaking in the left-right twin Higgs.

\section{ACKNOWLEDGMENTS}

We thank Zackaria Chacko for valuable discussions and comments on the draft. $\mathrm{C}$. $\mathrm{K}$ is supported by the NSF under grant PHY-0801323. H.S.G is supported by the NSF under grant 
PHY-04-57315 and by the DOE under grant DE-AC02-05CH11231.

[1] R. Barbieri and A. Strumia, arXiv:hep-ph/0007265.

[2] N. Arkani-Hamed, A. G. Cohen and H. Georgi, Phys. Lett. B 513, 232 (2001)

[3] N. Arkani-Hamed, A. G. Cohen, E. Katz, A. E. Nelson, T. Gregoire and J. G. Wacker, JHEP 0208, 021 (2002); N. Arkani-Hamed, A. G. Cohen, E. Katz and A. E. Nelson, JHEP 0207, 034 (2002); T. Gregoire and J. G. Wacker, JHEP 0208, 019 (2002); I. Low, W. Skiba and D. Smith, Phys. Rev. D 66, 072001 (2002) D. E. Kaplan and M. Schmaltz, JHEP 0310, 039 (2003).

[4] H. C. Cheng and I. Low, JHEP 0309, 051 (2003) [arXiv:hep-ph/0308199]; H. C. Cheng and I. Low, JHEP 0408, 061 (2004) [arXiv:hep-ph/0405243]; H. C. Cheng, I. Low and L. T. Wang, arXiv:hep$\mathrm{ph} / 0510225$.

[5] H. Georgi and A. Pais, Phys. Rev. D 10, 539 (1974); Phys. Rev. D 12, 508 (1975).

[6] D. B. Kaplan and H. Georgi, Phys. Lett. B 136, 183 (1984); D. B. Kaplan, H. Georgi and S. Dimopoulos, Phys. Lett. B 136, 187 (1984); H. Georgi and D. B. Kaplan, Phys. Lett. B 145, 216 (1984).

[7] Z. Chacko, H. S. Goh and R. Harnik, Phys. Rev. Lett. 96, 231802 (2006) [arXiv:hep-ph/0506256];

R. Barbieri, T. Gregoire and L. J. Hall, arXiv:hep-ph/0509242. Z. Chacko, Y. Nomura, M. Papucci and G. Perez, JHEP 0601, 126 (2006) [arXiv:hep-ph/0510273].

[8] Z. Chacko, H. S. Goh and R. Harnik, JHEP 0601, 108 (2006) [arXiv:hep-ph/0512088];

[9] H. S. Goh and C. A. Krenke, Phys. Rev. D 76, 115018 (2007) [arXiv:hep-ph/0707.3650].

[10] J. C. Pati and A. Salam, Phys. Rev. D 10, 275 (1974); R. N. Mohapatra and J. C. Pati, Phys. Rev. D 11, 566 (1975); R. N. Mohapatra and J. C. Pati, Phys. Rev. D 11, 2558 (1975); G. Senjanovic and R. N. Mohapatra, Phys. Rev. D 12, 1502 (1975).

[11] H. S. Goh and S. Su, Phys. Rev. D 75, 075010 (2007) [arXiv:hep-ph/0611015].

[12] A. Davidson and K. C. Wali, Phys. Rev. Lett. 59, 393 (1987); S. Rajpoot, Mod. Phys. Lett. A 2, 307 (1987) [Erratum-ibid. A 2, 541 (1987 PHLTA,B191,122.1987)].

[13] D. Chang and R. N. Mohapatra, Phys. Rev. Lett. 58, 1600 (1987).

[14] K. m. Cheung, Phys. Lett. B 517, 167 (2001) [arXiv:hep-ph/0106251]; T. Appelquist, B. A. Dobrescu and A. R. Hopper, Phys. Rev. D 68, 035012 (2003) [arXiv:hep-ph/0212073].

[15] E. M. Dolle and S. Su, Phys. Rev. D 77, 075013 (2008) [arXiv:hep-ph/0712.1234].

[16] A. Abada and I. Hidalgo, Phys. Rev. D 77, 113013 (2008) [arXiv:hep-ph/0711.1238].

[17] M. Gell-Mann, P. Ramond and R. Slansky, in Supergravity (North-Holland, Amsterdam, 1979);

T. Yanagida, in Proc. Workshop on Unified Theories and Baryon Number in the Universe;

R. N. Mohapatra and G. Senjanovic, Phys. Rev. Lett. 44, 912 (1980).

[18] W. Y. Keung and G. Senjanovic, Phys. Rev. Lett. 50, 1427 (1983); T. Han and B. Zhang, Phys. Rev. Lett. 97, 171804 [arXiv:hep-ph/0604064]; A. Atre, T. Han, S. Pascoli and B. Zhang, arXiv:0901.3589 
[hep-ph].

[19] O. Adriani et al. [L3 Collaboration], Phys. Lett. B 295, 371 (1992).

[20] P. Achard et al. [L3 Collaboration], Phys. Lett. B 517, 67 (2001) [arXiv:hep-ex/0107014].

[21] J. D. Vergados, Phys. Rept. 361, 1 (2002) [arXiv:hep-ph/0209347]; M. Doi, T. Kotani and E. Takasugi, Prog. Theor. Phys. Suppl. 83, 1 (1985).

[22] C. Arnaboldi et al., arXiv:0802.3439 [hep-ex]; H. V. Klapdor-Kleingrothaus et al., Eur. Phys. J. A 12, 147 (2001) [arXiv:hep-ph/0103062].

[23] D. W. Jung and J. Y. Lee, arXiv:hep-ph/0701071; Y. B. Liu, H. M. Han and Y. H. Cao, arXiv:hepph/0703268; Y. B. Liu and J. F. Shen, arXiv:hep-ph/0704.0840; Y. B. Liu, H. M. Han and Y. H. Cao, arXiv:hep-ph/0701169; D. W. Jung and K. Y. Lee, arXiv:hep-ph/0701087; W. Ma, C. X. Wei and Y. Z. Wang, arXiv:hep-ph/0905.0597; C. X. Yue, H. D. Yang and W. Ma, arXiv:hep-ph/0903.3720; Y. B. Liu, Y. H. Cao and H. M. Han, Eur. Phys. J. C 53, 615 (2008) [arXiv:hep-ph/0701169].

[24] A. Pukhov et al., arXiv:hep-ph/9908288; A. Pukhov, arXiv:hep-ph/0412191.

[25] J. Alwall et al., JHEP 0709, 028 (2007) [arXiv:0706.2334 [hep-ph]].

[26] G. Aad et al. [The ATLAS Collaboration], arXiv:0901.0512 [hep-ex].

[27] G. Abbiendi et al. [OPAL Collaboration], Eur. Phys. J. C 29, 463 (2003) [arXiv:hep-ex/0210031]. 